\title{
Research on Dynamic Simulation of Underwater Vehicle Manipulator Systems
}

\author{
Qiang $\mathrm{Li}^{1,2} \quad$ Qifeng Zhang ${ }^{1} \quad$ Xiaohui Wang ${ }^{1}$ \\ ${ }^{1}$ State Key Laboratory of Robotics, Shenyang Institute of Automation, Chinese Academy of Sciences \\ Shenyang China \\ ${ }^{2}$ Graduate School of the Chinese Academy of Sciences \\ Beijing China \\ \{liqiang,zqf,wxh\}@sia.cn
}

\begin{abstract}
This paper presents a simulator to simulating two key problems-underwater perception and autonomous manipulation。In the simulator a controller-observer strategy is proposed. A new SLAM (Simultaneous Localization and Mapping) algorithm-UKF (Unscented Kalman Filter) is used to estimate the state of UVMS (Underwater Vehicle Manipulator Systems) and the target object. 3D Simulation results show that the new SLAM algorithm can get the optimal estimation of state of UVMS and the target object, and the end-effector of manipulator can reach the desired object and the accuracy is centimeter level.
\end{abstract}

Keywords: UVMS, UKF, SLAM, position estimation, autonomous manipulation

\section{INTRODUCTION}

The development of underwater intervention technology can greatly increase the mankind's capability to understand, utilize and exploit the ocean. So in the field of ocean science research, energy sources, military and underwater engineering, the scientists and engineers are devoting themselves to increase the intervention capability of underwater vehicle. UVMS is a kind of underwater device with intervention capability, and able to use the manipulator mounted on the vehicle to complete the special underwater task. The task can include retrieving the geological specimens and install underwater instruments such as seismometers.

Although currently the most UVMS are operated by the pilot on the vessel, the autonomous and semi-autonomous is the development trend. Aiming at the autonomous operation capability of the UVMS, two research issues are proposedperception and autonomous manipulation. 1) Perception problems can be roughly divided into two types according to the information about the state of target objects and the UVMS. If the object is stationary, it is a mapping problem. About this subject SLAM and relative position estimation are two active areas. If the object is dynamic, it becomes a target motion estimation problem. About this subject, a great deal of research has been focused on the BOT(bearings-only tracking). 2) Autonomous manipulation is based on the information

Supported by “ 863” Program of China (2006AA09Z217). Supported by the Knowledge Innovation Program of Chinese Academy of Sciences (07A6050601). obtained in the stage of perception. The manipulator is driven to touch the target object. In this community, the coordinated motion planning and control is an active area. Although many approaches about motion planning and control of land-based and space robots have been discussed, these methods are not applicable to the UVMS. Because the base of UVMS is floating compared with the land-based robots and complex and uncertain hydrodynamics is forced on the UVMS compared with space robots.

In this paper by fusing the information from IMU(Inertial Measurement Units) and monocular camera, a map about UVMS and the target object is constructed in the unstructured environment. UVMS completes the manipulation task based on the map. The whole simulation research is finished on Matlab. The remainder of paper is arranged as follows. In section II, the related researches are summarized. In section III, difficulties, which will appear in the research about this paper, are presented, and the simulator's principle and strategy are proposed. In section IV a new method to solve the problem about SLAM is proposed. In section $\mathrm{V}$ motion planning of end-effector of manipulator is described. In section VI the simulation about map construction and manipulation is proposed to show that the new algorithm about SLAM is effective and the autonomous manipulation is successful.

\section{RELATED RESEARCH}

Many researches have been addressed on the perception vision-based in the field robots community. H.H.Wang [1] proposed an object-sensing system based on the stereo-vision. Jiang Wang [2] proposed 3D relative position and orientation estimation by the method of vision-based only. Not only position of target object but also its orientation is obtained in this paper. The estimation algorithm adopted in this paper is EKF. Huster[3] proposed the scheme of fusing information from IMU and camera, and demonstrated the underwater autonomous manipulation on a land-based manipulator. This paper is enlightened by Huster's research results. But there are much difference between them. 1) A new observer is proposed based on the kinematics. However the observer designed by Huster is based on the vehicle's dynamic and need the information about the thruster force. The advantage of new observer proposed in this paper is that it is separated with 
dynamics of vehicle and becomes a feedback component independent on the thruster force, which makes it more eligible to be used on all sorts of field robots. 2)Huster thought the perception problem as a relative position estimation problem, and only the relative position information is obtained. However in this paper the perception problem becomes a SLAM problem and all state of target object and UVMS are obtained. In this situation, once the map has been constructed even the object disappears from the view field, the UVMS can operate based on the map.

Many researches have been done about the SLAM in the field robots. Williams and Newman[4] proposed autonomous underwater simultaneous localization and map building based on the sonar. Kim[5] and Langelaan[6] applied the SLAM to the UAV(Unmanned Aerial Vehicle). Yufeng Liu[7] used the SLAM on the ground vehicle.

In the area of the autonomous manipulation, Professor Antonelli[8][9] investigated many aspects about UVMS, which included inverse kinematics resolution to find suitable vehicle/joints trajectory that correspond to a desired endeffector, adaptive tracking control of UVMS and interaction control of UVMS. Mclain[10] developed the hydrodynamic model of underwater manipulator in Standford University. Qifeng Zhang[11] researched the motion planning and control on UVMS and demonstrated the manipulation based on the simulation and pool experiment.

\section{Simulator Principle and Control Strategy}

IMU can provide the measurement value about the linear acceleration and the angular velocity of UVMS, and the monocular camera can provide the measurements of the bearing of the target object relative to the UVMS. Based on the information a map of target object can be constructed and the position and velocity of UVMS within the map can be obtained. This can be thought of as a SLAM problem. Since the first paper[12] about SLAM was proposed, EKF(Extended Kalman Filter) became the standard estimation algorithm to the nonlinear state and observing equations. However with regard to the problems occurred in this paper, EKF can not complete the estimation task. The reason is listed as follows.1) in the conventional SLAM problems, the information about range and bearing (eg. in [4])can be got. However, in this situation, only bearing of target is provided. 2) low-cost IMU and camera generate the measurement value with high noise level. 3) discretizing the continuous state and observing equations with Euler method introduces the unmodeled error. In this paper, UKF is implemented as solution to the three challenges.

In the paper a controller-observer strategy is adopted to complete the perception and autonomous manipulation task in the closed loop control system. The perception and manipulation scene is shown in Fig.1. The simulator principle is shown in Fig.2.

In Fig 1, two coordinate systems are mentioned: the inertial frame $(O X Y Z)$ and the body frame $\left({ }^{v} o^{v} x^{v} y^{v} z\right)$. The origin

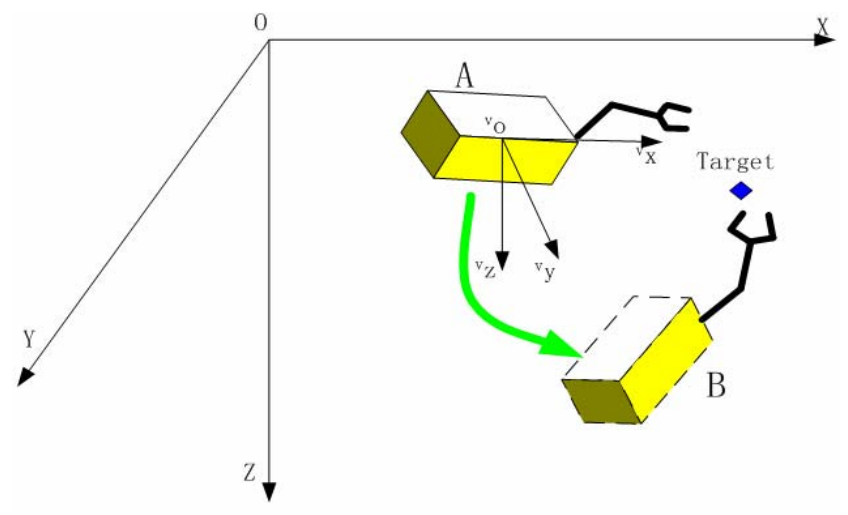

Fig. 1 Geometry of perception and manipulation

of the inertial frame is defined as the point where UVMS is located at the beginning of initial observing instant. The origin of the body frame is located in the point denoted as $\{X, Y, Z\}$ in the inertial frame, and the target object is denoted as $\left\{X_{0}, Y_{0}, Z_{0}\right\}$ in the inertial frame.

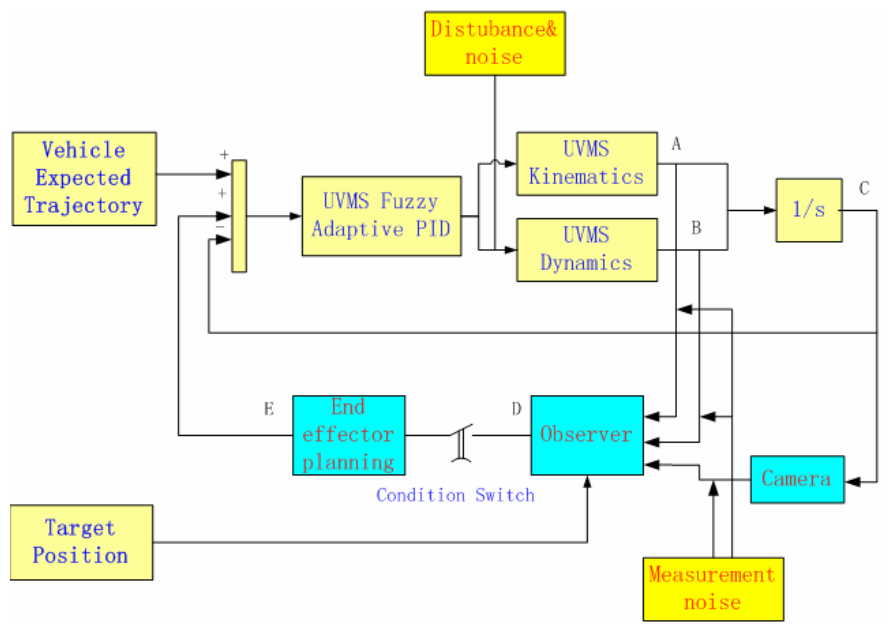

Fig. 2 Integrated Simulator Scheme

This simulator is a digital simulation system without actual sensors (camera and IMU) connected with the simulator. In order to produce the vision and linear acceleration and rotational velocity data which are obtained by sensors in actual manipulation, we introduce a actual nonlinear and timevarying kinematics and dynamics of UVMS to generate these data[11]. With noise added, these data can act as the actual data of sensors.

Position A represents the linear velocity, rotational velocity of vehicle and joint angular rate of manipulator in inertial frame, and it is denoted as $\left\{\dot{X}, \dot{Y}, \dot{Z}, \dot{\phi}, \dot{\theta}, \dot{\psi}, \dot{\theta}_{1}, \dot{\theta}_{2}\right\}$. Position $\mathrm{B}$ represents the linear acceleration and angular acceleration of the vehicle and the joint angular acceleration in the body frame, and it is denoted as $\left\{\dot{u}, \dot{v}, \dot{w}, \dot{p}, \dot{q}, \dot{r}, \dot{q}_{1}, \dot{q}_{2}\right\}$. Position D represents the estimation state of vehicle and position of target object and it is denoted as $\{X, Y, Z$, $\left.u, v, w, p, q, r, \alpha_{x}, \alpha_{y}, \alpha_{z}, b_{a x}, b_{a y}, b_{a z}, b_{p}, b_{q}, b_{r}, X_{0}, Y_{0}, Z_{0}\right\}$ 
where $\left\{\alpha_{x}, \alpha_{y}, \alpha_{z}, b_{a x}, b_{a y}, b_{a z}, b_{p}, b_{q}, b_{r}\right\}$ is differential of bias which will be described in detail in section IV. Position E represents the motion planning of manipulator's joint angle to reach the target object and it is denoted as $\left\{\theta_{1 e}, \theta_{2 e}, \dot{q}_{1 e}, \dot{q}_{2 e}\right\}$.Condition switch is a judging condition whether the observer has obtained the true relative position. If the condition is true, the relative position is fed back. The motion planning begins to compute the expected joint angle and angle rate to drive the manipulator to approach the target object. Otherwise the manipulator keeps static.

Because the camera is the unique sensor to provide the bearing information about target, the motion trajectory of UVMS has an importance effect on the observability of state. There are several trajectories which can be selected shown as Fig. 3

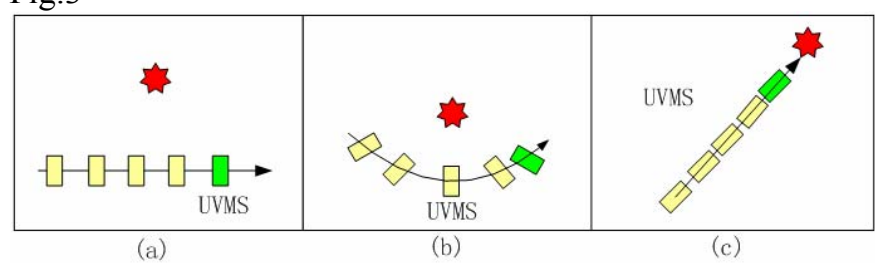

Fig 3 the Vehicle's Observing Trajectory

The movement trajectory described in (c) is not feasible, because no new bearing information is measured if the vehicle moves like that. The trajectory like (b) is not easy to realize for a real UVMS. So at the first research stage, the linear motion (a) is selected as the motion trajectory of UVMS. In fact, the motion planning is an important research issue. The planning principles are: 1) minimizing the uncertainty of state of UVMS and the target object. 2) minimizing the time or power to reach the object. Much work has been done in [13].

\section{State Estimation}

State estimation is based on the measurements from IMU and camera. So the first step in this part is constructing the model of IMU and the camera. The model will occur as the observing equations.

\section{A. Camera Model}

In order to approximate the real UVMS, the camera is located at the head of UVMS, and the offset between GC(gravity center) of vehicle and the OC(optical center) of camera is not neglected. The optical axis of camera is consistent with $\mathrm{x}$-axis of vehicle, and the effect focal length of camera is $f=1$. The camera is represented by the standard perspective projection model as shown in Fig 4.

The vehicle's coordinate frame is $\left({ }^{v} o^{v} x^{v} y^{v} z\right)$ and the camera coordinate frame is $\left({ }^{c} O^{c} x^{c} y^{c} z\right) . T\left(X_{0}, Y_{0}, Z_{0}\right)$ is the target object in the inertial frame, and $M\left(z_{y}, z_{z}\right)$ is the image point of object projected on the image plane. $O_{1}\left({ }^{v} X,{ }^{v} Y,{ }^{v} Z\right)$ is the position of GC of UVMS in the inertial frame. $O_{2}\left({ }^{c} X,{ }^{c} Y,{ }^{c} Z\right)$ is the position of $\mathrm{OC}$ of camera in the inertial frame. The image point can be described as follows:

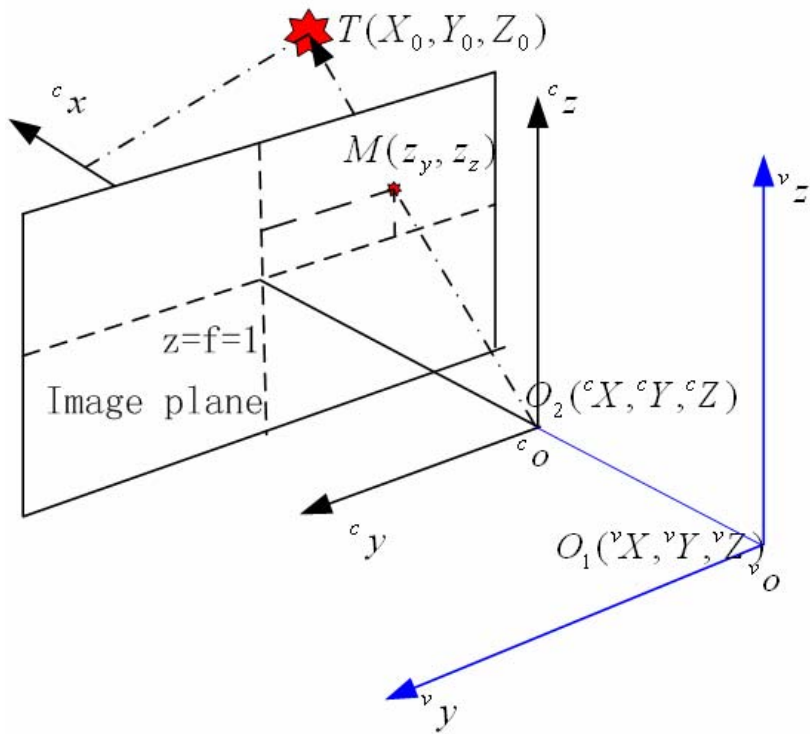

Fig. 4 Perspective Project Camera Model

$$
\begin{aligned}
z_{y} & =f^{* c} S_{y} /{ }^{c} S_{x}+n_{z y} \\
z_{z} & =f^{* c} S_{z} /{ }^{c} S_{x}+n_{z z}
\end{aligned}
$$

where $n_{z y}$ and $n_{z z}$ are the zero-mean white Gaussian noise. ( $\left.{ }^{c} S_{x},{ }^{c} S_{y},{ }^{c} S_{z}\right)$ is the projection of relative position between object and GC on the camera frame. It can be derived from $\left({ }^{v} S_{x},{ }^{v} S_{y},{ }^{v} S_{z}\right)$-- the projection of relative position between object and GC on the body frame.

$$
\left[\begin{array}{c}
{ }^{c} S_{x} \\
{ }^{c} S_{y} \\
{ }^{c} S_{z}
\end{array}\right]={ }_{v}^{c} T\left[\begin{array}{c}
{ }^{v} S_{x} \\
{ }^{v} S_{y} \\
{ }^{v} S_{z}
\end{array}\right]+\left[\begin{array}{l}
l_{x} \\
l_{y} \\
l_{z}
\end{array}\right]
$$

$\left(l_{x}, l_{y}, l_{z}\right)$ is the offset between the GC and OC. ${ }_{v}^{c} T$ is the transforming matrix from the body frame to the camera frame. In this paper it is $3 \times 3$ identity matrix.

$$
\begin{gathered}
{\left[\begin{array}{c}
{ }^{v} S_{x} \\
{ }^{v} S_{y} \\
{ }^{v} S_{z}
\end{array}\right]={ }_{n}^{v} T\left[\begin{array}{c}
X_{0}-{ }^{v} X \\
Y_{0}-{ }^{v} Y \\
Z_{0}-{ }^{v} Z
\end{array}\right]} \\
{ }_{n}^{v} T=\left[\begin{array}{ccc}
c \psi c \theta & s \psi c \theta & -s \theta \\
-s \psi c \phi+c \psi s \theta s \phi & c \psi c \phi+s \phi s \theta s \psi & c \theta s \phi \\
s \psi s \phi+c \psi c \phi s \theta & -c \psi s \phi+s \theta s \psi c \phi & c \theta c \phi
\end{array}\right]
\end{gathered}
$$

where ${ }_{n}^{v} T$ is the transforming matrix from the inertial frame to the body frame. $(\phi, \theta, \psi)$ is used to describe the roll-pitchyaw. $s(\cdot), c(\cdot)$, and $t(\cdot)$ mean $\sin (\cdot), \cos (\cdot)$ and $\tan (\cdot)$ respectively

\section{B. IMU Model}

IMU includes linear accelerometers and rate gyros. Egziabher[14] developed the model of accelerometer and gyro, here the results are given:

$$
z_{a}=\operatorname{diag}(\alpha)\left[\frac{d}{d t} a-T g\right]+b_{a}+n_{a}
$$




$$
z_{\omega}=\omega+b_{\omega}+n_{w}
$$

where $\alpha$ is the accelerometer scale factor and represented by $\operatorname{diag}(\alpha) \approx I_{3 \times 3}, \mathrm{~T}$ is the transforming matrix from the inertial frame to the body frame. $z_{a}, z_{\omega}$ are the measurement value of accelerometers and gyros. $b_{a}, b_{\omega}$ are sensor bias and can be thought of as random walk models.

$$
\begin{aligned}
& \dot{b}_{a x}=n_{b a x} \\
& \dot{b}_{a y}=n_{b a y} \\
& \dot{b}_{a z}=n_{b a z} \\
& \dot{b}_{\omega}=n_{b \omega}
\end{aligned}
$$

the scale factor can be modeled as

$$
\begin{aligned}
& \dot{\alpha}_{x}=n_{o x} \\
& \dot{\alpha}_{y}=n_{o y} \\
& \dot{\alpha}_{z}=n_{o z}
\end{aligned}
$$

$n_{a}, n_{\omega}$ are zero mean white Gaussian noise. $n_{b a x}, n_{b a y}, n_{b a z}$,

$n_{b \omega}, n_{\alpha x}, n_{o y}, n_{\alpha z}$ are zero mean white Gaussian noise.

$$
\begin{aligned}
& b_{a}=\left[b_{a x}, b_{a y}, b_{a z}\right] \\
& \alpha=\left[\alpha_{x}, \alpha_{y}, \alpha_{z}\right] \\
& b_{\omega}=\left[b_{p}, b_{q}, b_{r}\right]
\end{aligned}
$$

\section{Vehicle Kinematics}

A standard vehicle kinematics which is driven by inertial measurement is shown as follows:

$$
\begin{gathered}
\dot{X}=\cos \psi \cos \theta u+(\cos \psi \sin \theta \sin \phi-\sin \psi \cos \phi) v \\
+(\cos \psi \sin \theta \cos \phi+\sin \psi \sin \phi) w \\
\dot{Y}=\sin \psi \cos \theta u+(\sin \psi \sin \theta \sin \phi+\cos \psi \cos \phi) v \\
+(\sin \psi \sin \theta \cos \phi-\cos \psi \sin \phi) w \\
\dot{Z}=-\sin \theta u+\cos \theta \sin \phi v+\cos \theta \cos \phi w \\
\dot{\phi}=\left(z_{p}-b_{p}\right)+\tan \theta \sin \phi\left(z_{q}-b_{q}\right) \\
-\tan \theta \cos \phi\left(z_{r}-b_{r}\right) \\
\dot{\theta}=\cos \phi\left(z_{q}-b_{q}\right)-\sin \phi\left(z_{r}-b_{r}\right) \\
\dot{\psi}=\frac{\sin \phi}{\cos \theta}\left(z_{q}-b_{q}\right)+\frac{\cos \phi}{\cos \theta}\left(z_{r}-b_{r}\right) \\
\dot{u}=\frac{z_{x}}{\alpha_{x}}+g \sin \theta-b_{\rho x}-b_{a x}-n_{a x} \\
\dot{v}=\frac{z_{y}}{\alpha_{y}}-g \cos \theta \sin \phi-b_{\rho y}-b_{a y}-n_{a y} \\
\dot{w}=\frac{z_{z}}{\alpha_{z}}-g \cos \theta \cos \phi-b_{\rho z}-b_{a z}-n_{a z}
\end{gathered}
$$

$\left[z_{x}, z_{y}, z_{z}\right]$ is the measurement value of accelerometer. $\left[z_{p}, z_{q}, z_{r}\right]$ is the measurement value of gyro. $\left[n_{a x}, n_{a y}, n_{a z}\right]$ is zero mean white Gaussian noise. $\left[b_{\rho x}, b_{\rho y}, b_{\rho z}\right]$ is the acceleration item induced by the offset of IMU from the GC. It can be described as:

$$
\begin{aligned}
b_{\rho x}= & \left(\varepsilon_{y} \rho_{z}-\varepsilon_{z} \rho_{y}\right)+\left(q\left(p \rho_{y}-q \rho_{x}\right)\right. \\
& \left.-r\left(r \rho_{x}-p \rho_{z}\right)\right) \\
b_{\rho y}= & \left(\varepsilon_{z} \rho_{x}-\varepsilon_{x} \rho_{z}\right)+\left(r\left(q \rho_{z}-r \rho_{y}\right)\right. \\
& \left.-p\left(p \rho_{y}-q \rho_{x}\right)\right) \\
b_{\rho z}= & \left(-\varepsilon_{x} \rho_{y}-\varepsilon_{y} \rho_{x}\right)+\left(p\left(r \rho_{x}-p \rho_{z}\right)\right. \\
& \left.-q\left(q \rho_{z}-r \rho_{y}\right)\right)
\end{aligned}
$$

where $\mathcal{E}=\left[\mathcal{E}_{x}, \mathcal{E}_{v}, \mathcal{E}_{z}\right]$ is the angular acceleration. $\rho=\left[\rho_{x}, \rho_{y}, \rho_{z}\right]$ is the offset between the IMU and the GC of the vehicle.

Until now, the state equations and observing equations are constructed. State equations include Eqs. 5 11,15 23 and the observing equations are Eqs. 1 2.

\section{Unscented Kalman Filter}

The basic idea of UKF is that it's easier to approximate a probability distribution than to approximate an arbitrary nonlinear function or transformation [15]. UKF uses a set of points which are selected so that their mean and covariance are the current mean and covariance. The nonlinear function is applied the points, in turn, to yield a cloud of transformed points. The statistics of the transformed points can be calculated to form an estimate of the nonlinear transformed mean and covariance. Based on the idea, in the process of time update, a set of points are chosen according to the mean and covariance of the current optimal state estimation, then the points are applied to the iterative process model to obtain a clouds of transformed points. When the transformed points are generated, the weight values are produced, which are used to calculating the mean and covariance of transformed points. The same process occurs in the observing equation, except for the initial points are produced by the predicted mean and covariance. Like conventional Kalman filter, the filter gain is calculated and then the next time optimal state value is obtained.

The low cost IMU induces bias and random noise errors, which can lead to unbounded errors in the integrated quantities. Hence sensor bias must be included in the state vector. In this SLAM problem we choose the state variables as :

where

$$
x=\left[x_{v}, x_{o}\right]
$$

$x_{v}=\left[X, Y, Z, \phi, \theta, \psi, u, v, w, \alpha_{x}, \alpha_{y}, \alpha_{z}, b_{x}, b_{y}, b_{z}, b_{p}, b_{q}, b_{r_{T}}\right]^{T}$ represents the state of UVMS and $x_{0}=\left[X_{0}, Y_{0}, Z_{0}\right]^{T}$ represents the state of the object.

The state equations (kinematics-Eqs 15 23; IMU modelEqs 5 11) can be discretized into a general form by the Euler method. 


$$
\left[\begin{array}{c}
x_{v, k+1} \\
x_{o, k+1}
\end{array}\right]=f\left[\begin{array}{c}
x_{v, k}, z_{k} \\
x_{o, k}
\end{array}\right]+\left[\begin{array}{c}
w_{k} \\
0
\end{array}\right]
$$

where $w_{k}$ is zero mean white Gaussian noise. The unmodeled error induced by Euler method is included in it. $z_{k}$ is the measurement value from gyros and accelerometers.

\section{Time update}

According to Eq 27, when the initial state and the IMU measurements are given and the angular acceleration is obtained from the dynamics model[11], the one step predicted value can be got.. Another two important items which should be attention are the covariance of initial state and the process covariance. It should be set as possible as less in order to guarantee the converging of the algorithm.

\section{Measurement update}

The observing equations (Eqs.1 2) can be discretized into a general form

$$
\left[\begin{array}{c}
z_{y, k} \\
z_{z, k}
\end{array}\right]=g\left({ }^{c} S_{x, k},{ }^{c} S_{y, k},{ }^{c} S_{y, k}\right)+v_{k}
$$

where $v_{k}$ is the zero mean white Gaussian noise.

The frequency of time update and measurement update has great effect on the performance of algorithm. Usually the frequency of IMU is faster than vision. At the simulation stage, a worst condition is used, that is, the frequency of IMU and the frequency of vision are all equal to the sample frequency of vision.

\section{E. Planning of End-effector Trajectory}

On the UVMS, which the paper is discussing, a two link manipulator is used to perform the task of manipulation on the horizontal plane. Under the condition of moving of vehicle, the observer can estimate the position of the vehicle and the target object. So the UVMS is able to extend its manipulator to approach the target while the vehicle is moving. Two steps need to be taken to perform the task. Firstly, an expected trajectory, in which the end effector will move from the start point to the target, is planed. Secondly the trajectory needs to be transferred from the task space to the joint space. This is a inverse kinematics problem. The manipulation in horizontal plane is shown in Fig 5. When vehicle is located in B, the manipulator is in $\mathrm{C}$, the trajectory is planed from $\mathrm{C}$ to $\mathrm{D}$ in task space and joint space.

In the body frame, the position of end effector and the elbow joint is calculated:

$$
\begin{gathered}
x_{\text {end }}=x_{\text {off }}+L 1 * \cos \theta_{1}+L 2 * \cos \left(\theta_{1}+\theta_{2}\right) \\
y_{\text {end }}=y_{\text {off }}+L 1 * \sin \theta_{1}+L 2 * \sin \left(\theta_{1}+\theta_{2}\right) \\
x_{j o \text { int }}=x_{o f f}+L 1 * \cos \theta_{1} \\
y_{j o \text { int }}=y_{\text {off }}+L 1 * \sin \theta_{1}
\end{gathered}
$$

where $\left(x_{\text {end }}, y_{\text {end }}\right)$ is the position of end effector, $\left(x_{\text {off }}, y_{\text {off }}\right)$

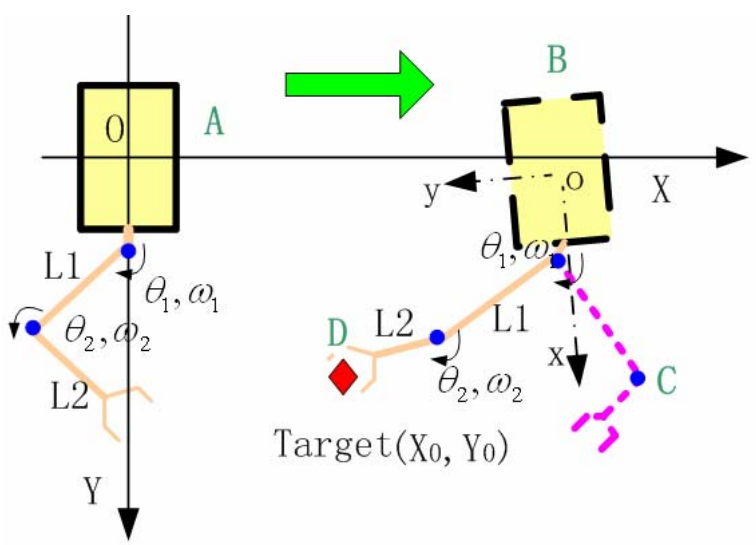

Fig .5 the Geometry of UVMS Manipulation

is the position of manipulator's base, and $\left(x_{j o \text { int }}, y_{j o \text { int }}\right)$ is the position of elbow joint position.

Apparently, from the Eqs. 29 32, the joint trajectory can not be calculated according to the position of end effector. The velocity equations are got by differentiating the Equations.

$\dot{x}_{\text {end }}=-L 1 * \omega_{1} * \sin \theta_{1}-L 2 *\left(\omega_{1}+\omega_{2}\right) \sin \left(\theta_{1}+\theta_{2}\right)$

$\left.\dot{y}_{\text {end }}=L 1 * \omega_{1} * \cos \theta_{1}+L 2 *\left(\omega_{1}+\omega_{2}\right) * \cos \theta_{1}+\theta_{2}\right)$

so, the angular rate of joints can be obtained:

$$
\left[\begin{array}{c}
\omega_{1} \\
\omega_{2}
\end{array}\right]=\left[\begin{array}{cc}
\left.-L 1 * \sin \theta_{1}-L 2 * \sin \theta_{1}+\theta_{2}\right) & \left.-L 2 * \sin \theta_{1}+\theta_{2}\right) \\
\left.L 1 * \cos \theta_{1}+L 2 * \cos \theta_{1}+\theta_{2}\right) & \left.L 2 * \cos \theta_{1}+\theta_{2}\right)
\end{array}\right]^{-1} *\left[\begin{array}{c}
\dot{x}_{\text {end }} \\
\dot{y}_{\text {end }}
\end{array}\right]
$$

Let

$$
\begin{aligned}
& \dot{x}_{\text {end }}=k_{x} *\left(x_{\text {tar }}-x_{\text {end }}\right) \\
& \dot{y}_{\text {end }}=k_{y} *\left(y_{\text {tar }}-y_{\text {end }}\right)
\end{aligned}
$$

$k_{x}$ and $k_{y}$ are the coefficients. $\left(x_{t a r}, y_{t a r}\right)$ is the position of target object in the horizontal plane.

$$
\begin{aligned}
& x_{\text {tar }}=X_{0}-X \\
& y_{\text {tar }}=Y_{0}-Y
\end{aligned}
$$

If the initial joint angles are known, the trajectory planning algorithm of joint angles and the end effector are given above.

\section{SimUlation Result}

In this part two simulations are implemented. The first one is constructing the map to demonstrate the effective of UKF to SLAM problem. The other is autonomous manipulation simulation and the simulation result shows that the accuracy can be up to centimeter level.

\section{A. Constructing Map}

A linear motion trajectory is selected when the vehicle observes the target to construct map. If we select the point where the vehicle begins to move, that is, the algorithm begins to run, as the origin of the inertial frame, the map can be constructed. Here we assume the vehicle moves from $(0,0,0)$ to $(3,0,2)$. The real position of target is located at $(1,1.5,1.5)$. The initial position and orientation of UVMS can be set $x_{v, 0}=[0,0,0,0,0, p i / 2,0,0,0,1,1,1,0,0,0,0,0,0]$,and the 
initial object state is generated at the run time. It is a random vector which is described as

$x_{o, 0}[1+0.5 *$ rand, $1.5+0.5 *$ rand, $1.5+0.5 *$ rand $]$ rand means a random number between -1 to 1 . Because in the underwater environment the visionable distance is about $1 \sim 3 \mathrm{~m}$, the selection of this vector abides with the real underwater environment. Here the random number is used, which shows the initialization of object is stochastic in a certain range. The stochastic initial position demonstrates that the initial value has a little effect on the observer. The initialization of state covariance is selected as possible as less. The quantities level is $10^{-8}$ except that the initial covariance of position of the target is $0.5^{2}$. Measurement covariance is

$$
\sigma_{z y}^{2}=0.01^{2}, \sigma_{z z}^{2}=0.01^{2} \text {. }
$$

The trajectory of vehicle and the map are shown in Fig.6.

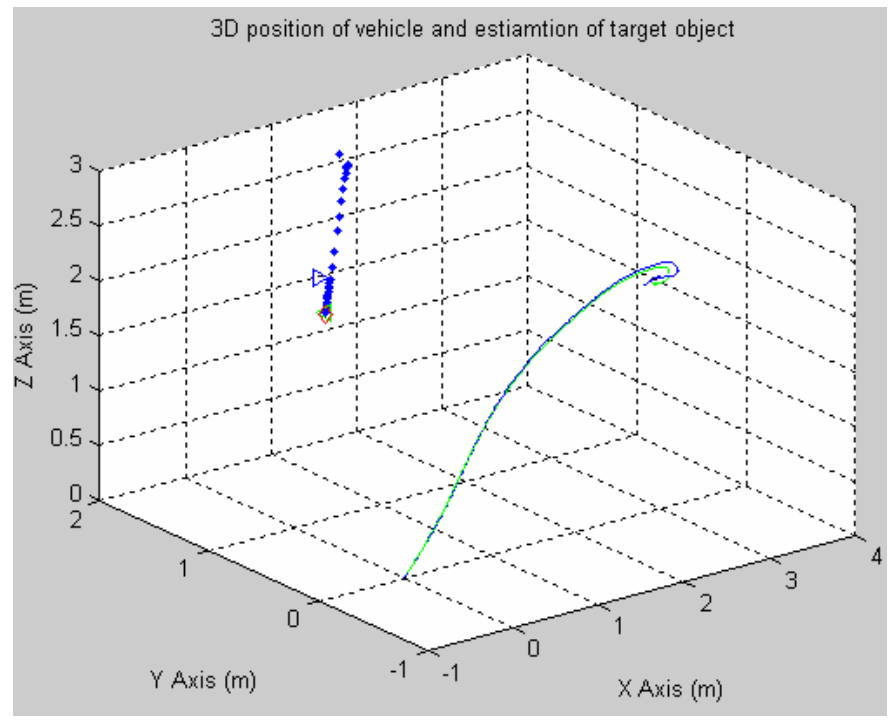

Fig. 6 Map of SLAM Problem

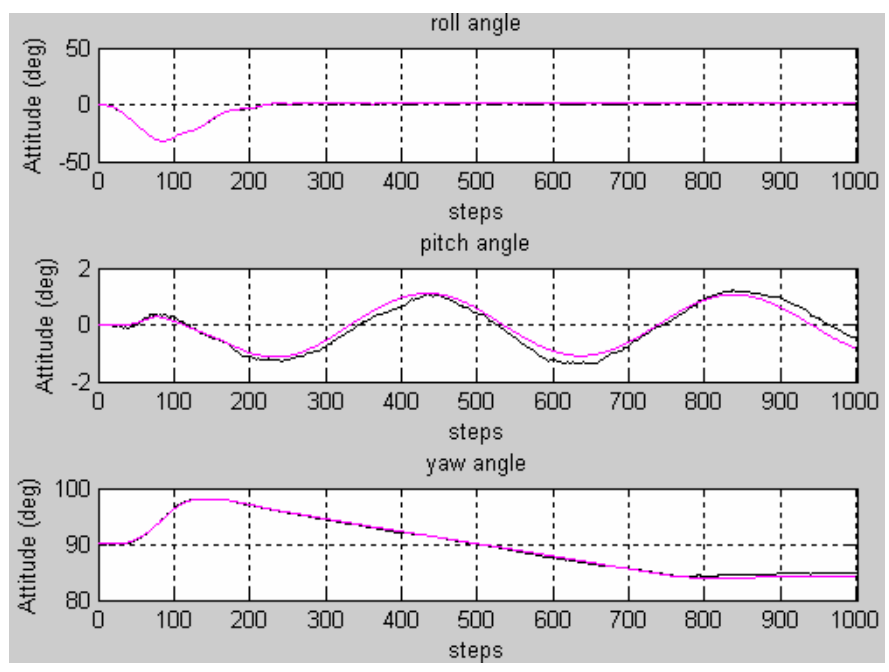

Fig.7 comparison in attitude of vehicle

The green line is the real vehicle's motion trajectory, and the blue line is the estimation of vehicle's trajectory. The triangle of blue is the initial position of the target object. The green triangle is the ultimate estimation position. The red diamond is the real target position. We can see that the green triangle is consistent with the red diamond, so observer estimates the position of object successfully.

Fig 7 shows the comparison between the real attitude of the vehicle and the attitude's estimation value obtained from the observer (red represents the real value, and the black represents the estimation value).

\section{B. Autonomous Manipulation}

In this simulation, the vehicle moves in the horizontal plane, from $(0,0,0)$ to $(2,0,0)$. The length of vehicle is $2 \mathrm{~m}$, the length of joint 1 connected with vehicle is $1 \mathrm{~m}$ and the second joint's length is $0.3 \mathrm{~m}$. In Fig. 8 half of vehicle is shown, and in the body frame, the target is moving and the manipulator is driven to approach the target. The red square represents the start point when the manipulator begins to move and the green diamond represents the end point of target when the simulation is finished.

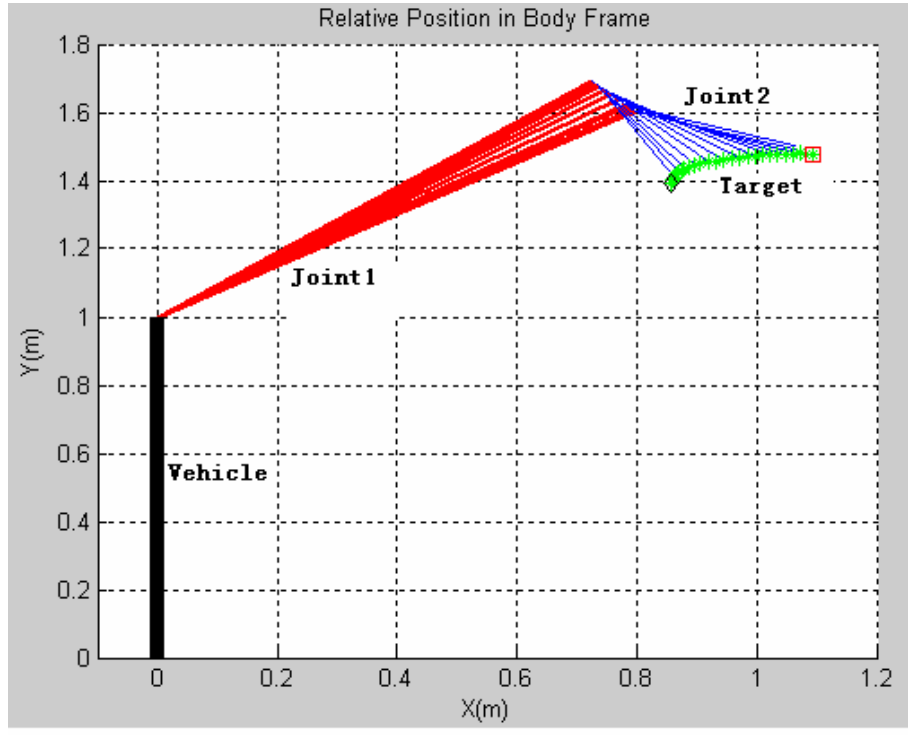

Fig. 8 Scene of manipulator's touching the target point

The result shows that at last the end-effector can reach the target, and the accuracy is centimeter-level.

\section{Discussion}

The noise level of sensors(IMU and Camera) and the sample period have effect on the performance of observer. Simulation results show that too large noise level and too low sample frequency can cause the observer divergence. In this paper a worst sample frequency-30hz is used, which most low cost vision sample system and IMU can reach this level. The covariance of accelerometer used in the simulation is $0.01^{2}$ and the angular velocity covariance is $0.008^{2}$, the camera covariance is $0.01^{2}$. The low-cost sensors can reach the quantities level.

Because the discretization induces the unmodeled error, the initial state covariance and process noise covariance can not be obtained accurately. That means no accurate prior 
knowledge can be got for the SLAM problem. So the initial value is set with a "seemed reasonable" value. The simulation results show that the setting method is feasible.

\section{CONCLUSION}

This paper proposes a simulator to simulate the perception and manipulation for the UVMS. An Observer is used to estimate the state of UVMS and the target object, by which a map is constructed. A controller and observer strategy is used to realize the automatic manipulation.

Simulation can demonstrate that the observer's algorithm is feasible and provide the sensor's noise level which is necessary for the convergence of observer.

\section{ACKNOWLEDGMENT}

The authors wish to acknowledge the support for this work provided by the Underwater Research Center, Shenyang Institute of Automation, thank for Doctor Qifeng Zhang's software support about UVMS system, and thank for the syntax correction from Miss Wenping Qiu.

\section{REFERENCES}

[1] Howard H. Wang, "Experiments in Intervention autonomous underwater vehicles, "phd thesis, Stanford University 1996.

[2] Jiang Wang, William J.Wilson, "3D Relative position and orientation estimation using kalman filter for robot control," Proceedings of the 1992 IEEE International Conference on Robotics and Automation, May 1992

[3] Huster, "Relative position sensing by fusing monocular vision and inertial rate sensors," Phd thesis, Stanford University, 2003

[4] Stefan B.Williams, Paul Newman and Hugh Durrant-Whyte, "Autonomous underwater simultaneous localization and map building," Proceedings of IEEE International Conference on Robotics and Automation, 22-28 April 2000, San Francisco CA, USA
[5] Jong-Hyuk Kim and Salah Sukkarieh, "Airborne simultaneous localization and map building," Proceedings of the 2003 IEEE International Conference on Robotics \& Autonomous. Taipei,Taiwan, 2003

[6] Jack Langelaan and Steve Rock, "Navigation of small UAVs operating in forests," AIAA Guidance,Navigation, and control Conference and Exhibit, 16-19 August 2004,

[7] Yufeng Liu and Sebastian Thrun, "Results for outdoor-SLAM using sparse extended information filters," Proceedings of the 2003 IEEE International Conference on Robotics \& Autonomous, Taipei, Taiwan, 2003

[8] Gianluca Antanelli and Stefano Chiaverini, "Task-Priority Redundancy Resolution for Underwater Vehicle-Manipulator Systems," Proceeding of the 1998 IEEE International Conferrence on Robotics \& Automation, Leuven,Belgium, May 1998

[9] Gianluca Antonelli, "Underwater Robots Motion and Force Control of vehicle-Manipulator Systems," Springer Tracts in Advanced Robotics Volume 2, Sringer-Verlag Berlin Heidelberg, New York,2003

[10] Timothy W. Mclain, "Modeling of underwater manipulator hydrodynamics with application to the coordinated control of an arm/vehicle system," PhD thesis, Stanford University, 1995

[11] Qifeng, Zhang, "Research on coordinated motion planning and control of autonomous underwater vehicle-manipulator system," $P h D$ thesis, Graduate School Chinese Academy of Sciences, 2007

[12] R. C. Smith and P. Cheeseman, "On the respresentation and estimation of spatial uncertainty," International Journal of Robotic Research, 4(5), 1986.

[13] Tarun Kanti Podder, "Motion planning of autonomous underwater vehicle-manipulators system," Phd thesis, University of Hawaii,2000

[14] Demoz Gebre-Egziabher, "Design and performance analysis of a lowcost aided dead reckoning navigator," PhD thesis, Standford University, 2004.

[15] Simon J.Julier and Jeffery K.Uhlmann, "Unscented filtering and nonlinear estimation," IEEE Review, vol. 92, no. 3, 2004 